\title{
If You Build it Will They Come? Patients, Providers and Blockchains in Health Technology Assessment
}

\author{
Paul C Langley, PhD, Adjunct Professor, College of Pharmacy, University of Minnesota MN
}

Robert E Martin, MS, RPh, Folsom CA

\begin{abstract}
It is an open question as to whether blockchains can become an integral part of health care management in the US. On the one hand, there are the advocates of blockchains who see them as empowering patients to capture property rights to their medical records in a secure, encrypted, and portable form. On the other hand, there are blockchain critics that see the opportunities offered in health care as little different from those offered in other industries, viewing a blockchain structure as one that may reduce administrative and transaction costs, with little thought given to the potential of blockchain platforms to support a range of health technology assessment activities. While previous commentaries have pointed to this potential, the obstacles offered by the absence of clearly defined property rights and the absence of a market for DNA profiles have not been explored. The case put forward here is that any expectation that a blockchain as a 'one-stop-shop' for the interrogation of personal health records alone is unlikely to succeed. Apart from property rights effectively blocking this business model the blockchain vendor should consider targeted value added activities. At best, only a subset of records has the possibility of being transferred, with ongoing concerns regarding their quality and scope. This does not mean that the blockchain software model should be rejected. Far from it. The blockchain as a health technology assessment platform has the potential to support added value activities which not only improve the process of care and reduce costs and improve efficiencies, but also provide an ideal framework for property rights assignment. This opens the door to incentives and the monetization of value added health data by patients and providers, capturing rents that are at the moment expropriated by third parties. Critical issues are not only property rights and creation of a market place, but the ability to link and incentivize patients and their providers to support active blockchains to generate value added.
\end{abstract}

Keywords: blockchain, success criteria, technology assessment platform, DNA property rights

\section{Introduction}

Will blockchain technology transform US health care? Will the presumptive claims for blockchains as platforms to support health technology assessment, be recognized and adopted by patients, providers and health care systems? To what extent will the opportunity presented by blockchains, as opposed to existing and widely adopted patient record software systems, support the potential for property rights transfer and the marketing of health data under smart contracts to third parties?

The purpose of this commentary is to consider whether there is market potential for blockchains as a software platform to support health technology assessment? Recently published commentaries in INNOVATIONS in Pharmacy have explored the potential role of blockchains as one software platform option in such assessments ${ }^{1,2,3}$. The arguments presented in these commentaries pointed to an unmet need for the evaluation and replication in target patient populations of claims for new and existing products. The purpose of this commentary is to argue that the benefits of blockchain technology are more likely to be achieved if the vendor, not only has an appropriate

\section{Corresponding author: Paul C Langley, PhD}

Adjunct Professor

College of Pharmacy University of Minnesota

Director, Maimon Research LLC

5061 North Apache Hills Trail, Tucson, AZ 85750

Email: langley@maimonresearch.com management structure in place to support data access and monetization, but that the vendor adopts an active rather than a passive model of data assembly.

\section{A Secure Medical Record}

To date, there seems little evidence as to whether or not there is a demand for a secure, portable and readily accessible blockchain driven medical record. Rather, it seems to have been assumed: (i) that patients are dissatisfied with current medical record storage and access protocols and (ii) that there is a groundswell of opinion in favor of mandating the transfer of medical record ownership to patients. Given these assumptions, the advocates of blockchains have then leapt to the conclusion that, following experience and proposed innovations in banking and finance with their specific demands for security, encryption, validated transactions and access, blockchains are the obvious solution for a medical record 'one-stop-shop'. The retail aura being supported by the presumed ability and interest of patients to monetize their medical record by offering smart contracts to third parties.

If our criteria are for a secure yet portable and accessible on demand medical record repository, then blockchains are certainly a solution. There is, however, the need to distinguish a blockchain as a secure record repository from a blockchain as an active platform that can not only provide a secure transaction ledger for medical encounters but support value added interventions and health technology assessment protocols; a 
transaction ledger which can not only record medical encounters but can support commercially viable research and assessment activities.

Once the question of value added activities in health technology assessment are brought into the picture, then we can consider moving beyond the passive blockchain model as a lifetime record of encounters within a range of medical environments current and previous provider primary care visits, referrals, urgent care visits, emergency room visits and hospitalizations to one where the elements of a medical record available through the blockchain are only one part of a platform that can capture additional real-time data elements through disease specific value added activities. With property rights assigned, these complementary data elements can then offer the promise of smart contracting and monetization.

\section{Some Pertinent Questions}

A number of questions have to be addressed if this vision of an active blockchain is to be realized. Who owns the medical record? Are property rights a potential barrier to effective record transfer and aggregation over target patient populations? How do we transfer grandfathered medical records that may go back over decades? Is the data transfer protocol for these various medical locations applicable to them all? What permissions would have to be obtained? Does the blockchain capture in one time-stamped verified transaction text, video and scans (and even billing) for an entire encounter such as a two-day hospitalization? How do we judge the quality of the data that are transferred to the blockchain? Does the blockchain support effective medical record search protocols? Can it include misdiagnoses that are later revisited? Are the transaction ledgers complete? Does the blockchain include the complete list of prescribed pharmaceuticals with brand/generic names, dosage, formulation number of tablets and National Drug Code? Are there precise markers such as ICD-10-CM codes and CPT codes? Does the blockchain include an algorithm to translate prior ICD-9-CM codes to ICD-10-CM codes (the latter mandatory from February 2014)? Does the blockchain provide links to ICD-10-CM text titles and whether these are billable codes?

Apart from raising questions as to the coverage of a blockchain medical record, the quality of the data and the completeness of the record, any attempt to identify specific data elements may involve an unacceptable search time as each prior locked transaction (and there will be hundreds if. If the blockchain is seen only as a portable yet passive record, is it able to support medical emergencies outside of the patient's primary care environment? Will the attending physician and staff in an emergency room be able to review the medical record in time? More to the point: what does the blockchain offer over and above present admission protocols (e.g., screen-based questionnaires with default algorithms) which are designed to accommodate admission vitals and record the visit? What evidence is there that access by a treating physician at a remote site to the patient's blockchain record will materially improve outcomes?

Certainly, blockchains are an elegant solution. But this is only a presumption; is it a solution in search of a problem? We need to think more carefully as to what blockchains offer, obstacles to their endorsement by health systems, patients and providers, and the contribution they can make to unmet needs in health technology assessment. After all, if the rallying cry is for record ownership and monetization, a reasonable question for patients is the likely return from data ownership. If it is only in the tens of dollars it may be less than attractive.

\section{Blockchain Recruitment}

If we think about a vendor focused on developing a viable blockchain product, then the first question to ask is: how are patients to be recruited to the blockchain? There are two avenues: a patient can enroll unilaterally, and then request the blockchain vendor to facilitate transfer of their medical records to the blockchain, or the blockchain vendor can contract with a provider to act as an intermediary in recruiting practice patients to the blockchain. This latter recruitment avenue is to be preferred, as it not only supports a more rapid and focused recruitment of patients, but that it also sets the stage for future value-added technology assessment activities that jointly involve patient and provider.

Unless the provider wishes to be actively involved in technology assessment, the contribution of blockchains as a monetized record resource will be limited. If the focus is on creating blockchain value through investing in health technology assessment activities, then the provider is a critical part of the ability to capture and monetize the assembled data. Certainly, the provider may facilitate transfer of medical records to the blockchain, but these records may be, at best, a secondary source of data. If the focus within specific disease areas is, for example, response to therapy, there are more than likely a number of endpoints not captured in the medical record. At the same time, searching the medical record for endpoints may be frustrating, not only due to the lack of standardized responses, but the difficulties of trying to identify responses through search algorithms.

A continuing focus in health technology assessment is mining of de-identified patient records. While there is substantial literature demonstrating the ability to draw substantive conclusions on therapy impacts from de-identified medical records (e.g., the UK Clinical Practice Research Datalink and its earlier incarnation as the General Practice Research Database $^{4}$ ) the limitation is these data are assembled after the data bus has left the depot. The blockchain platform allows, to extend the metaphor, value added activities in data assembly to 
populate the bus with 'a better class' of passenger. It is possible to generate a database of de-identified records and apply search and artificial intelligence to, literally, tease out responses. A far more robust approach is to see the blockchain as a vehicle to support structured mining of therapy response with inputs from both the provider and the patient.. This allows, not only for agreement on the assignment of property rights between the provider and patient but a firm basis for monetization; a structured interrogation of data that have been quality assessed.

\section{Property Rights}

A question which has not been addressed fully in the blockchain literature is the role of property rights to medical records. There are clearly expectations by a number of blockchain developers that blockchains have a role as a secure yet portable lifetime repository for a patient's medical records; a medical record 'one-stop-shop'. Certainly, blockchains offer this potential. Unfortunately, the question of property rights to those records may torpedo this 'one-stop-shop' vision. The question then arises: if blockchains are able only to capture and monetize through smart contracts medical records to which the patient has undisputed property rights, is it possible to consider blockchains as a software platform with a wider yet complementary role, a role which also allows smart contracting and monetization?

Certainly, there are widespread concerns over the security of medical records. Instances abound on hacking of systems and the ransoming of re-access, with the UK National Health Service hospital data a well-established and ongoing target. The fact that security can be breached and patient medical records put at risk is not, in itself, a justification for a jump to blockchain software as the 'one size fits all' solution. There are a number of unresolved questions. Are we to consider only the security of patient health records? Is there a demand by health systems to move to a blockchain solution for record storage (scalability issues)? Are blockchains to be seen only as a way of reducing health system administrative and transaction costs? United Health care seems to take this point in proposing a pilot project to control better provider records and contacts in an insurance system. Should a health care system capture individual patient encounters in a blockchain? Does this mean we will have two blockchains: one for health systems (and individual providers) that captures stores, encrypts, validates and timestamps all patient encounters in that system, possibly linked to their electronic medical record software system, with another blockchain for individual patients? Consider a matrix of encounters (the individual cells of the matrix) with health providers. The rows of the matrix are defined for individual patients while the columns capture the health systems where encounters may occur. Under the health system blockchain, each column of the matrix represents the encounters of patients with the particular provider while the rows capture the encounters of an individual patient with the various providers. This latter row entry defines the electronic medical record or the capture of records by the individual patient's blockchain. Each record element of the provider-patient matrix has property rights. These may be unambiguously assigned to the patient or provider, or may be in a 'fuzzy' limbo where property rights are yet to be adjudicated. If these row data elements, for each patient, are attempted to be transferred to the blockchain but are blocked or delayed, then the data set may be incomplete and fragmented.

In single payer health systems in countries such as Australia, New Zealand and the UK the property rights to medical health records are typically claimed by the government. If blockchains are to be advocated, then the patient's rights would be limited. Unless there was an agreement to surrender property rights in favor of the patient, then there would be no opportunities for smart contracting and monetization. More to the point, unless lifetime record security and portability were considered a concern by both patients and system managers, there would be no incentive to abandon the present software environment.

Interestingly, the focus on who owns whose data in our blockchain ecosystem has been on the patient, versus the provider or health system. Once the matrix picture is considered, the property rights scope opens to the possibility of conflict over property rights between providers. Does, for example, a primary care provider (PCP) who refers a patient to a cardiologist, have property rights over the cardiologist's record? Including, even, the summary report typically sent to the PCP? Can the PCP assume property rights and, without reference to the cardiologist, transfer these to the patient? All challenging questions that need to be addressed and answered!

As noted in previous commentaries, there is no national agreement in the US on medical record property assignment ${ }^{1,2}$. This is within the jurisdiction of individual states. New Hampshire is the only state that has assigned property rights to the patient; outside of New Hampshire some 21 jurisdictions have property rights assigned to the provider, with the added complication that patients typically assign property rights to their provider, with providers also assigning their rights to insurers and other potential vendors of medical data. Given this, and the reluctance of many health systems to relinquish rights in which they may have a monetary interest with sales to third parties, it would be unwise for a blockchain developer to assume that a patient has undisputed property rights. As most developers have limited resources, it is unlikely that they would have the inclination to address this issue. They may live in the hope that the federal government might intervene and override state and commercial interests. This is unlikely.

Unfortunately, the absence of agreed and well-defined property rights to the records that make up a patient's health history means, irrespective of any technical issues associated with 
record transfer and search, that the vision of a blockchain medical record capturing a patient's complete medical history and transforming this into a viable commercial product is unlikely to be realized. Not only would the record set be incomplete, there would be no necessary concordance between patients over which data elements from which provider are actually captured in the individual blockchains. The more practical barrier to a 'complete' blockchain record is the presence of substantial commercial interests in stand-alone health records. Undoubtedly, there would be pushback on property rights assignment and monetization; an ongoing debate that would be unlikely to be resolved in the near term. Claims that patients have an inherent right to their records, and the prospects that ownership may support monetization, may simply be ignored.

The attraction of challenging property rights and eliminating erecting barriers to market entry to protect revenue streams can be seen in the case of DNA profiles. There are now a number of vendors for DNA riding on the back of interests in family genealogy. Typically, an individual will submit a specimen 'spit', this is analyzed and a report sent to the client. The cost can range from the low to mid hundreds of dollars. Clients are given, in some cases, the option of allowing access to their deidentified DNA profile by third parties. In the case of 23andMe, a leading vendor, a recent agreement with GlaxoSmithKline (GSK), a leading pharmaceutical company, was for the transfer of millions of DNA records for the sum of $\$ 300$ million. Under this business model the client pays for the DNA profile (it covers the costs of production) while contributing to the vendors bottom line (Profit line 1). In selling the DNA profiles to GSK, 23andMe establishes Profit line 2. At no stage is the client informed of potential monetization of their DNA profile. The revenue stream from GSK is pure rent. In abrogating property rights, the client is out of pocket. First, through meeting the costs of production of the DNA and contributing to the vendors bottom line while, second, failing to capture any part of the rent accruing from GSK to the vendor.

The client loses out because of the abrogation of property rights. The rights accrue to the DNA vendor who can extract as much rent as possible through contracting the DNA profiles (perhaps multiple times for each profile, to different third parties). The client has no idea of revenue (or DNA royalties) forgone because of the absence of a market to give signals as to the price of DNA profiles (which may not all have the same market value). The only way to avoid this is for the client to insist on ownership of the DNA profile. The profile would be lodged on the blockchain with the DNA vendor confirming that the profile is not retained in their records. At the same time, the client should insist that the DNA vendor, and there may be competition between vendors, should pay the client for their profile. Once one DNA vendor offers to pay for profiles, the rest will fall into line. The only remote exception being where the vendor warrants not selling to third parties. The client contracts with the DNA vendor as agent for third party use with a market price established for sale to a third party to be determined, when the DNA vendor contracts with a third party. Again, if a market is established, third parties would be expected to compete for vendor contracting. The alternative scenario is where the blockchain vendor acts as agent for the individual in monetizing their DNA for third parties under smart contracts.

It is not surprising in the ancestor-focused world of DNA profiles, vendors attempt not only to retain property rights to client profiles, but simultaneously avoid provision of information on the imputed market value of the profile. This value may be derived from the 'mining of the profile' by a third party pharmaceutical manufacturer and potentially augmented if linked to other databases. Not surprisingly again are efforts by vendors such as 23 andMe, to set up artificial barriers to market pricing, profile dissemination and capture in platforms such as blockchains. Giving a concrete example, the imputed value or shadow price of a profile with nominated characteristics is $\$ 1,000$ and the special summer sale DNA profile offer is $\$ 100$ (with a profile production cost to the vendor of \$20) then the client gives away not only the $\$ 1,000$ of pure rent (less contracting costs) and any fee paid by the vendor to the patient for the right to undertake the DNA assay (which will vary with the profile). Under present arrangements, the return to the vendor is $\$ 1,080$ for the profile, while nothing accrues to the client.

\section{Value Added and the Blockchain Record}

If the concern is with the completeness of the medical record, then the scenario where the provider acts as the agent or intermediary in patient recruitment might actually facilitate transfer of property rights. The provider sees it is in the interests of both parties for medical records from the practice to be copied over to the blockchain and property rights recognized by both parties. This shared interest in generating as complete a blockchain record as possible can also extend to value added activities. As noted above, if disease specific platforms for assessing the value of competing drugs and devices are built, then there is the opportunity for patients and providers to co-operate in both evaluating the process of care and response to therapy, as well as facilitating monetization to third parties.

Unless property rights can be assigned and enforced through smart contracts, claims that patients can take control of their medical records, transfer them to a blockchain allowing them to monetize any record through smart contracts are likely nonstarters. Patients would only have only the ability to contract for those records where an unambiguous property right exists. This does not mean that they do not have the right to view their records and share these with other medical professionals as the need or emergency arises. In many instances this is already 
feasible, at least for the PCP record, which often includes records from referrals and encounters with urgent care, emergency rooms and hospitals. Some specialist providers now provide similar access, with hospitals not far behind. The point remains, however, that the records are fragmented and typically non-interoperable.

If property rights to the existing medical record (and records capturing future encounters) block the ability to create a blockchain record analogue, the blockchain vendor faces the risk of commercial failure. Patients may be unwilling to enroll in the blockchain if they see little chance of effective monetization of their records. The blockchain will fail to achieve a 'required' minimum number of enrollees to support, even with incomplete or fragmented medical records, viable commercialization. Potential third-party clients such as pharmaceutical manufacturers will have little interest in the data and opportunities to evaluate product performance.

Accepting the proposition that blockchains that rely exclusively on transferred medical records face an uncertain commercial future, the question then becomes one of whether the active blockchain business model is feasible. The blockchain model could support the creation of commercially viable data sets, with a reasonable probability of enrolling a minimum number of patients in a relatively short time frame to ensure commercial viability.

The appeal of the active blockchain model is that it provides the opportunity for the blockchain vendor to work with both the patient and the provider, while creating incentives structured for both participants, including the opportunity to generate additional practice revenue for the provider. Data collected in added value applications for chronic disease states, to give one example, would be structured to allow for ease of interrogation through data dictionaries. Data elements can be selected from the medical record and linked to the structured data, with the added advantage of access to a more complete medical record set for that particular application.

The viability of the value-added business model depends on a number of factors. First and foremost is to demonstrate the ability of the blockchain vendor, who will presumably be coordinating the value added data assembly, to meet not only required professional standards in health technology assessment but the ability to target providers and provider groups to demonstrate the benefits of a blockchain. Second, the blockchain vendor must demonstrate that the proposed software platform meets the required standards for data transfer, storage, smart contracting and monetization. Third, as the role of the proposed platform is to support patients and providers in the long term, there must be confidence in the ability of the vendor to provide such support. Finally, the vendor should have a viable business strategy to roll out the blockchain.
This is crucial, as the ability of the blockchain vendor to exploit the opportunities in health technology assessment (detailed in previous commentaries) have to be demonstrated to potential clients; not least of whom are providers and health system managers who may consider contracting, hopefully in the long term, with the a blockchain vendor.

\section{Conclusions: First Steps to a Viable Active Blockchain}

It would be foolish to think as noted in the title of this commentary, that patients, providers and health system managers will flock to a blockchain vendor. If the focus is on health technology assessment, previous commentaries have pointed to the range of assessment activities that could be undertaken with a 'mature' blockchain ecosystem in place in a health system. The challenge for a blockchain vendor is twofold: (i) targeting the most likely candidates for establishing a blockchain to support health technology assessment and (ii) demonstrating the viability of a blockchain in terms of its technical performance and its consequent ability to generate a substantive revenue stream, while demonstrating true downstream (outcomes, efficiency, cost savings, etc.) value for the client involved.

As described in previous commentaries, the blockchain should be seen as an umbrella (or exoskeleton) for supporting data generated from: (i) the transferred electronic medical records or links to the established record and (ii) from added value targeted interventions in specific disease states that generate additional data elements. The critical issue is the ability to generate data that not only support more effective care but also serve as a potential revenue source to third parties. This has yet to be demonstrated even though potential clients will see this as a sine qua non, not only of engaging with a blockchain system but of data transfer to that system. In summary, why go to the problem of dealing with property rights if the data elements that can eventually be transferred to generate the fragmented medical record into blockchain are impossible to identify and link to other data elements.

The creation of value added data goes to the heart of questions promoting a blockchains value in health technology assessment: the ability, within target patient groups to meet unmet medical needs in the comparative evaluation of healthcare interventions and the response of patients to treatment interventions in real world environments. There are any number of disease states where the data for medium to long-term response is either limited or absent. This would be the major selling point to health systems and providers: putting in place a platform which allows a more focused assessment of care, tracking outcomes, evaluating competing drugs and devices, while at the same time meeting quality standards mandated by assessment agencies.

Conflict of Interest: No conflicts were declared by either PCL or REM 


\section{References}

${ }^{1}$ Langley PC. Information or Evidence? Abandoning imaginary worlds for blockchains in health technology assessment. Innov Pharm. 2018;9(3): No.12.

${ }^{2}$ Langley PC, Martin RE. Blockchains, property rights and health technology assessment in the pharmaceutical and device(s) industries. Innov Pharm. 2018; 9(4): No.1

${ }^{3}$ Langley PC, Martin RE. Blockchains, formulary evaluations and health technology assessment. Innov Pharm. 2018;9(4): No. 2

${ }^{4}$ Clinical Practice Research Datalink https://www.cprd.com/home/ 